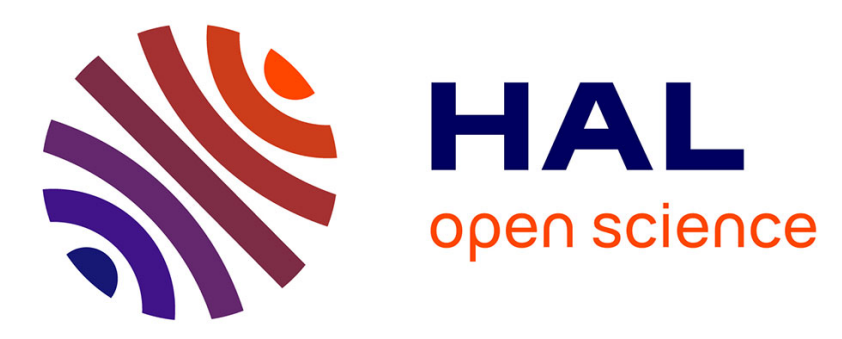

\title{
Dispersive Wave Interaction between Solitons in Fiber Lasers with Saturable Absorbers
}

Andrey Komarov, Foued Amrani, Alexander Dmitriev, Konstantin Komarov, Dmitry Meshcheriakov, François Sanchez

\section{- To cite this version:}

Andrey Komarov, Foued Amrani, Alexander Dmitriev, Konstantin Komarov, Dmitry Meshcheriakov, et al.. Dispersive Wave Interaction between Solitons in Fiber Lasers with Saturable Absorbers. Symposium on Photonics and Optoelectronics (SOPO), May 2012, Shanghai, China. 10.1109/SOPO.2012.6270437 . hal-03192910

\section{HAL Id: hal-03192910 \\ https://univ-angers.hal.science/hal-03192910}

Submitted on 8 Apr 2021

HAL is a multi-disciplinary open access archive for the deposit and dissemination of scientific research documents, whether they are published or not. The documents may come from teaching and research institutions in France or abroad, or from public or private research centers.
L'archive ouverte pluridisciplinaire HAL, est destinée au dépôt et à la diffusion de documents scientifiques de niveau recherche, publiés ou non, émanant des établissements d'enseignement et de recherche français ou étrangers, des laboratoires publics ou privés. 


\title{
Dispersive wave interaction between solitons in fiber lasers with saturable absorbers
}

\author{
A. Komarov, F. Amrani, A. Dmitriev, K. Komarov, D. Meshcheriakov, F. Sanchez
}

\begin{abstract}
On basis of numerical simulation it is found that the long-distance soliton wings can be formed by dispersive waves emitted by ultrashort pulses due to of various lumped elements in passive mode-locked fiber lasers. Peculiarities of the interaction of two solitons through such wings in lasers with lumped saturable absorbers (SA) are analyzed. Different sets of bound steady-states of two-soliton molecule are obtained. Among them there are sets with and without an alternation of parity of neighboring quantum steady-states. It is shown that the investigated interaction can result in mechanisms of both attraction and repulsion of loosely bound solitons. It is demonstrated a coding of an information by highly-stable bound soliton sequences.
\end{abstract}

Index Terms-Dispersive waves, bound solitons, passive modelocked fiber lasers.

\section{INTRODUCTION}

Passive mode-locked fiber lasers are widely used in many areas of science, technology, and engineering. Special place among the great diversity of lasing regimes belongs to multiple pulse passive mode-locking. A large amount of publications is devoted to this area of researches. Authors of the paper [1] experimentally demonstrated multihysteresis dependence of a number of pulses on pumping. The theory of this phenomenon was presented in the papers [2], [3]. Depending on properties of the interaction, the ultrashort pulses could either space themselves equally along a laser cavity, group into a tight bunch, or be more or less randomly distributed. In the experimental papers [4], [5] it was demonstrated that a set of several hundreds lasing solitons can form complexes analogical to various aggregate states of matter: a soliton gas, a liquid, a glass, a soliton crystal, a polycrystal. An elementary unit of any complex is a pair of interacting solitons. Bound steady-states of two solitons were investigated in papers [6] [9]. Steady-states with phase differences for peak amplitudes of solitons equal to $\pi, \pi / 2$ and 0 were predicted. The phase differences $\pi / 2$ and $\pi$ were experimentally observed [8], [9].

Possibility of a realization of strong bonds between interacting solitons $(\sim 10 \%$ of a single soliton energy) was numerically found in [10], [11] for erbium fiber laser with lumped losses due to a nonlinear polarization rotation technique. Spectrum of a single pulse has powerful sidebands that points out a possible role of dispersive waves emitted by solitons in the formation of strong intersoliton bonds. Previously

A. Komarov, F. Amrani, F. Sanchez are with the Laboratoire de Photonique d'Angers EA 4644, Université d'Angers, 2 Bd Lavoisier, 49000 Angers, France

A. Komarov, A. Dmitriev, D. Meshcheriakov are with the Novosibirsk State Technical University, K. Marx Pr. 20, 630092 Novosibirsk,Russia

K. Komarov, D. Meshcheriakov are with the Institute of Automation and Electrometry, Russian Academy of Sciences, Acad. Koptyug Pr. 1, 630090 Novosibirsk, Russia

Corresponding author: andrey.komarov@univ-angers.fr theoretical and experimental investigations had demonstrated the interaction of solitons through the sidebands resulting in a quantization of intersoliton separations in the soliton pair in such laser [12].

At the present time, much attention is given to a study of passive mode-locking of fiber lasers with SA based on various nanomaterials (media with quantum wells, nanotubes, graphene, etc.) [13], [14]. These lasers are described by simpler physical models than fiber lasers using the nonlinear polarization rotation technique. The simple models provide a way insight into the nature of both an interaction between solitons and established multisoliton regimes of passive modelocked fiber lasers.

In this paper we study an interaction of solitons through the dispersive waves due to a lumped SA and investigate lasing regimes realized under such interaction. In Sec. II we present a laser model for the study of the dispersive-wave mechanism of a formation of powerful soliton wings. Numerical simulation and discussion of the obtained results are given in Sec. III. In Sec. IV on basis of model of a passive mode-locked fiber laser with nonlinear polarization rotation technique it is demonstrated a coding of an information by highly-stable bound soliton sequences. The basic conclusions are made in Sec. V.

\section{LASER MODEL AND MASTER EQUATIONS}

The investigated laser is schematically represented in Fig. 1. It consists in a unidirectional ring cavity including a gain fiber

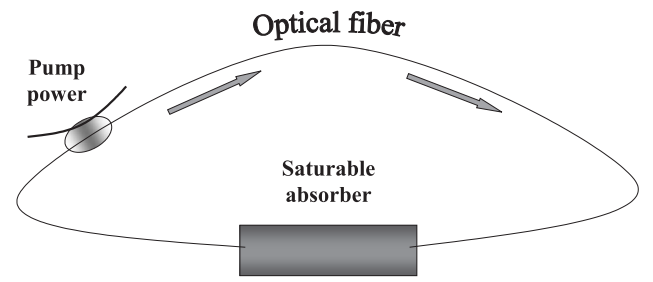

Fig. 1. Schematic representation of the studied passive mode-locked fiber laser.

medium and SA. The evolution equations for a field have the following form [3]

$$
\begin{gathered}
\frac{\partial E}{\partial \zeta}=\left(\mathrm{D}_{\mathrm{r}}+i \mathrm{D}_{\mathrm{i}}\right) \frac{\partial^{2} E}{\partial \tau^{2}}+\left(G+i q|E|^{2}\right) E, \\
E_{n+1}(\tau)=E_{n}(\tau) \exp \left(-\sigma_{0}-\frac{\sigma_{n l}}{1+p I_{n}(\tau)}\right),
\end{gathered}
$$

where $E(\zeta, \tau)$ is the field amplitude, $\tau$ is the time coordinate, $\zeta$ is the number of passes of radiation through the cavity, 
$D_{r}$ and $D_{i}$ are the frequency dispersions for gain-loss and for refractive index, respectively, $q$ is the Kerr nonlinearity, $G$ is the saturable amplification: $G=a /\left(1+b \int I d \tau\right), a$ is the pumping parameter, $b$ is the saturation one, $I=|E|^{2}$. Equation (2) determines the relation between the fields before and after $n$-th pass of radiation through SA. The value $\sigma_{0}$ is linear losses, $p$ is a saturation parameter for nonlinear losses, $\sigma_{n l}$ is their unsaturated value. Numerical simulation has been performed for typical parameters of Er-doped fiber laser with the anomalous dispersion of group velocity [4].

\section{NUMERICAL SIMULATION RESULTS AND DISCUSSION}

Figure 2 shows the distances between two interacting solitons in stable steady-states. There exist three typical structures of intersoliton separations. For the small separation, there is no alternation of bound states, there are only states with phase difference $\delta \varphi$ equal to $\pi$. For the medium separation, there exists the alternation of states with $\delta \varphi=\pi$ and 0 . For the large separation, there is no alternation of states again, and there are only states with $\delta \varphi=0$.

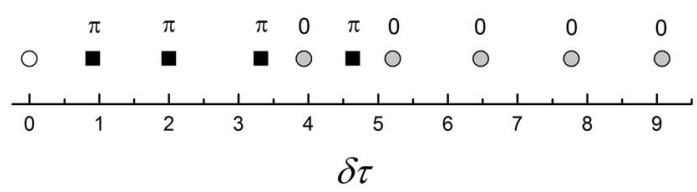

Fig. 2. Distances between two bound solitons in stable stationary states. The white circle corresponds to the first soliton. The black squares and grey circles do to the second soliton with the intersoliton phase differences $\delta \varphi=\pi$ and 0 , respectively. $a=0.5, \mathrm{D}_{\mathrm{r}}=0.01, \mathrm{D}_{\mathrm{i}}=0.1, q=1.5, p=1, \sigma_{0}=0.01$, $\sigma_{n l}=1$.

In the central part of the Fig. 2 one can see the structure with the alternation of odd and even states. Here the intersoliton separation is determined by a distance for which a phase change along the first soliton wing is equal to $\pi$. Such set of steady-states of a two-soliton molecule has been obtained in the numerical simulation of a fiber laser with a nonlinear polarization rotation technique [11]. The odd and even steadystates are described correspondingly by odd and even field functions $E(\tau)=\mp E(-\tau)$. The phase difference for the peak amplitudes of the solitons equals approximately $\pi$ for odd states and 0 for even states.

For small intersoliton separations the even states turned out unstable. As a consequence, the structure with only odd established steady-states is realized, contrariwise for large separations for which odd states are unstable (for both cases the intersoliton separations are determined by a distance with a phase change along the first soliton equal to $2 \pi$ ). We have also observed a simultaneous instability for both types of steady-states. In this case the interaction between solitons results in their attraction or repulsion. With varying nonlineardispersive parameters the sequence shown in Fig. 2 could change considerably.

Large quantity of steady-states and established intersoliton separations shown in Fig. 2 arises from interaction of ultrashort pulses through their powerful long-distance wings. The observed powerful sidebands in a single soliton spectrum point out the important role of dispersion waves in a formation of the soliton wings. The dispersive waves are emitted by solitons because of lumped SA. To test the role of the dispersive waves in the formation of the soliton wings we have used the following methodology. It is well known that a spatially uniform distributed nonlinear losses does not result in dispersive waves. In our analysis we use the model with the combination of distributed and lumped SA, which are described by the model of two-level absorber atoms [15]. The lumped part of

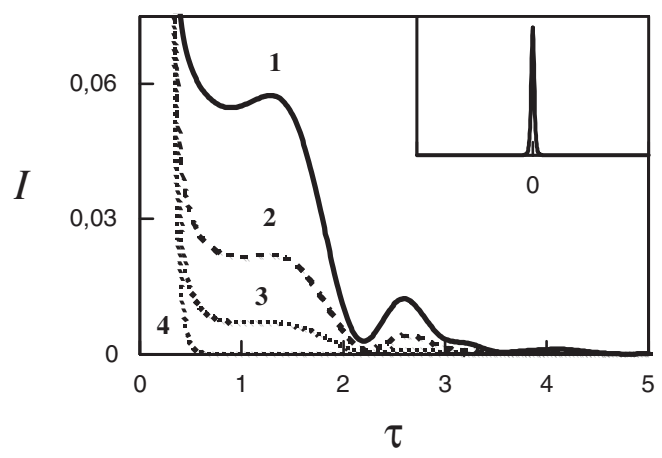

Fig. 3. Soliton wing intensity $I(\tau)$ under varying lumped fraction of SA $\eta$ : (1) $\eta=1$, (2) $\eta=0.75$, (3) $\eta=0.50$, (4) $\eta=0$. The other parameters are the same as in Fig. 2

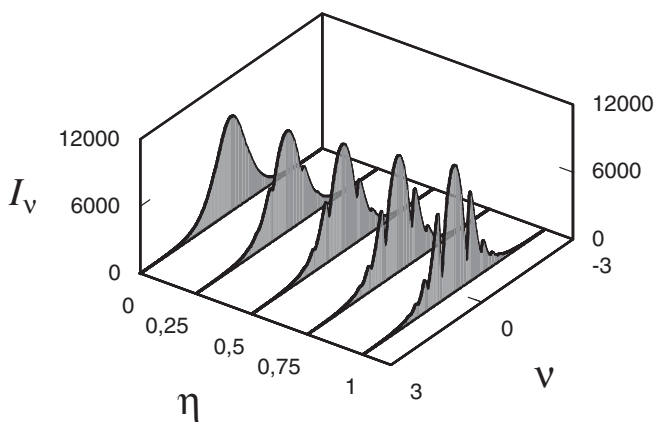

Fig. 4. Spectrum of single soliton with varying value of lumped fraction of SA $\eta$. The other parameters are the same as in Fig. 2

nonlinear losses is presented by Eq.(2) with the expression $\eta \sigma_{n l} /\left(1+p I_{n}\right)$ in the parenthesis. The distributed part of nonlinear losses is described by the additional expression $(1-\eta) \sigma_{n l} /\left(1+p I_{n}\right)$ in the second parentheses in Eq. (1). Varying the parameter $\eta$ from 1 to 0 we change the fraction of the lumped losses from 1 to 0 and respectively the fraction of the distributed ones from 0 to 1 . As this takes place, the total nonlinear losses remain unchanged and the central part of the individual soliton does not almost change. However its wings and spectrum change drastically as shown in Figs. 3, 4. With decreasing lumped fraction of nonlinear losses the soliton wings and spectral sidebands quickly weaken. Without lumped losses neither strong interaction between solitons nor set of large quantity of bound states (see Fig. 2) become possible. Use of model of distributed SA based on averaging of lumped SA losses, and also the account of finite length of lumped SA 
have led only to insignificant changes $(\sim 10 \%)$ in dependences shown in Figs. 3, 4.

We have also investigated the dynamics of formation of powerful wings through dispersive waves. Its main features are the following. In medium with a focusing Kerr nonlinearity and with an anomalous dispersion there exist stable selflocalized waves in the form of stationary conservative solitons. If any perturbation removes the soliton from a stationary state then it transients again into a stationary one through relaxation oscillations with a loss of the excess energy through emission of dispersion waves. The oscillation frequency is determined by the rate of a chirp formation resulting from nonlinearity and dispersion of the refractive index. The frequency dispersion of gain-losses results only in any additional peculiarities of this process. The soliton propagating in ring laser cavity experiences periodically shock perturbation when it passes through a lumped SA. Thus, the investigated system is analogical to a damped oscillator submitted to an external periodic force. In the established regime the structure of radiation is reproduced after each pass of a field through a laser cavity. However along a fibre the soliton parameters (peak intensity, duration, chirp, etc.) oscillate. Figure 5 shows the periodical change in the

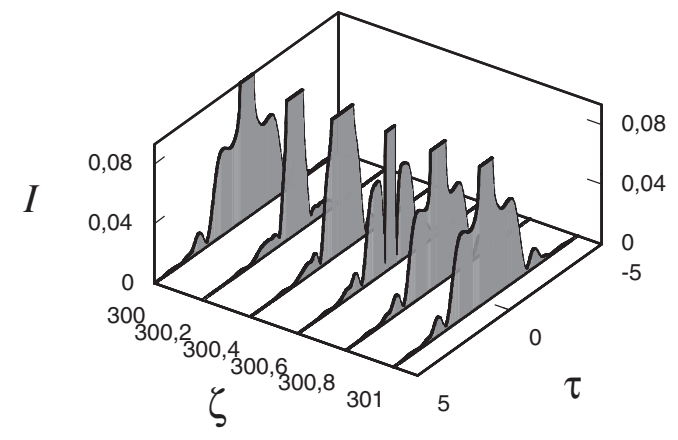

Fig. 5. Periodical change in a soliton pedestal during one pass through the laser cavity. The parameters are the same as in Fig. 2

soliton pedestal during a single pass through the laser cavity in the established operation.

For the pump $a \approx 0.42$ and $a \approx 0.55$ the oscillation period equals respectively one and half cavity round-trip period. Under such resonance the single soliton operation becomes unstable and transforms into the regime of two and three bound solitons, correspondingly. The pedestal structures differ cardinally for the pump above and below threshold values.

In Fig. 5 one can see the dispersive waves leaving from the soliton. These waves form the powerful long-distance soliton wings which result in spectral sidebands. In the case of normal dispersion, conservative solitons are not realized and consequently both powerful pulse wings and powerful spectral sidebands also are not realized.

Figure 6 shows the change in a phase along the soliton in established operation after each round-trip period. In aroundtrip the total phase change in any point of the pulse is multiple of $2 \pi$ ( $\delta \varphi=2 \pi n$, where $n$ is the integer). Each fragment of the wing is determined by a fixed $n$ which in turn determines a distance between neighboring inphase points at a soliton wing.

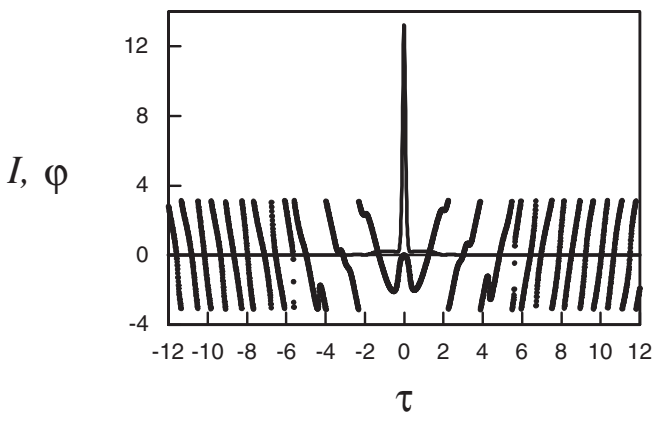

Fig. 6. Phase change $\varphi$ along the soliton wings. $\sigma_{0}=0.1$, the other parameters are the same as in Fig. 2.

For the central part of a pulse $n=0$, for the near wings $n=1$, for the far wings $n=2$.

\section{INFORMATION SEQUENCES OF BOUND SOLITONS}

Due to large binding energies, it is possible to realize highlystable noise-proof multisoliton molecules, that was numerically found in [10], [11] for erbium fiber laser with lumped losses due to a nonlinear polarization rotation technique. In this case the ground bound steady-state is characterized by minimal intersoliton distance and intersoliton phase difference equal to $\pi$, the first excited steady-state has the distance about two time bigger than minimal one and phase difference equal to 0 . Arranging several initial pulses on certain distances from each other, after transient process we can obtained stationary "molecular chains" with any desirable distribution of types of bonds between neighboring solitons along a pulse train. Such sequence is realized more simply with a use of the ground and first excited types of intersoliton bonds for which the binding energies are especially great. Distributing types of intersoliton bonds along a soliton train by required appointed order, it is possible to code an information. Figure 7 shows the

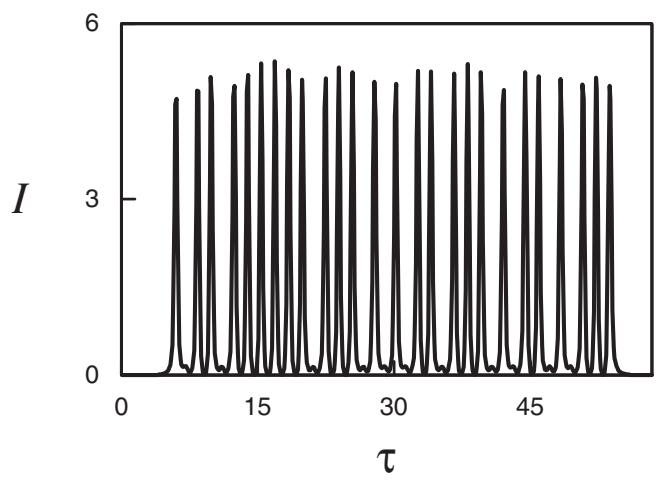

Fig. 7. Stable train of bound solitons with the ground and first excited $(\delta \varphi=0)$ types of bonds in which the number 21052012 is coded in binary system 1010000010011101001101100 . The smaller distance between pulses $(\delta \varphi=\pi)$ corresponds to 0 . The greater distance between pulses $(\delta \varphi=0)$ does to 1 .

example of information soliton sequence in which the number 
21052012 (corresponding 21.05.2012, the date of opening of the conference SOPO 2012) is coded in binary system. Here the ground and first excited types of a bond correspond to zero and to unit, respectively. In binary system this sequence corresponds to the number 1010000010011101001101100 . The transformation of this number from a decimal system to a binary one is following: $1 \cdot 2^{24}+0 \cdot 2^{23}+1 \cdot 2^{22}+0 \cdot 2^{21}+$ $0 \cdot 2^{20}+0 \cdot 2^{19}+0 \cdot 2^{18}+0 \cdot 2^{17}+1 \cdot 2^{16}+0 \cdot 2^{15}+0 \cdot 2^{14}+$ $1 \cdot 2^{13}+1 \cdot 2^{12}+1 \cdot 2^{11}+0 \cdot 2^{10}+1 \cdot 2^{9}+0 \cdot 2^{8}+0 \cdot 2^{7}+1 \cdot 2^{6}+$ $1 \cdot 2^{5}+0 \cdot 2^{4}+1 \cdot 2^{3}+1 \cdot 2^{2}+0 \cdot 2^{1}+0 \cdot 2^{0}=21052012$.

Such soliton trains are highly stable formations. The high stability is primarily due to large binding energies. Furthermore, there exists a second reason of the high stability. It consists in the following. The perturbation energy which was initially localized in the vicinity of some pair of bound solitons is quickly collectivized among all solitons of the train. In the numerical simulation we have used the random radiation noise to prove this stability. This noise induces up to $10 \%$ fluctuations of peak intensities of solitons but does not change the structure of soliton sequences.

\section{CONCLUSION}

On basis of numerical simulation we have found that the principal mechanism of formation of powerful long-distance soliton wings in laser with lumped SA is connected with dispersive waves emitted by soliton because of its interaction with lumped losses. These wings induce strong interaction between solitons leading to formation of soliton molecule with reach structure of energy levels and stable steady-states. It is shown that odd and even steady-states may have different degree of stability. As a result one can observe only odd neighboring states, or even ones, or states with an alternation of the parity. In the case of instability of both types of steadystates the intersoliton interaction can result in mechanisms of both attraction and repulsion of solitons. The bound soliton sequences can be used to code an information.

\section{ACKNOWLEDGMENTS}

The work is supported by the Agence Nationale de la Recherche (Contract ANR-2010-BLANC-0417-01SOLICRISTAL) and by the RF President's grant MK2602.2011.2.

\section{REFERENCES}

[1] D.Y. Tang, W.S. Man, and H.Y. Tam, "Stimulated soliton pulse formation and its mechanism in a passively mode-locked fibre soliton laser," Optics Commun., vol. 165, pp. 189-194, Jul. 1999.

[2] A.K. Komarov, and K.P. Komarov, "Multistability and hysteresis phenomena in passive mode-locked lasers," Phys. Rev. E, vol. 62, no. 6, pp. R7306-R7610, Dec. 2000.

[3] A. Komarov, H. Leblond, and F. Sanchez, "Multistability and hysteresis phenomena in passively mode-locked fiber lasers," Phys. Rev. A, vol. 71, pp. 053809, May 2005.

[4] F. Amrani, A. Haboucha, M. Salhi, H. Leblond, A. Komarov, and F. Sanchez, "Dissipative solitons compounds in a fiber laser. Analogy with the states of the matter," Appl. Phys. B, vol. 99, no. 1-2, pp. 107-114, Apr. 2010.

[5] F. Armani F., M. Salhi, H. Leblond, A. Komarov, F. Sanchez, "Passive harmonic mode locking of soliton crystals," Optics Letters, vol. 36, no. 21, pp. 4239-4231, Nov. 2011.
[6] N.N. Akhmediev, A. Ankiewicz, and J.-M. Soto-Crespo, "Multisoliton Solutions of the Complex Ginzburg-Landau Equation," Phys. Rev. Lett. vol. 79, no. 21, pp. 4047-4051, Nov. 1997.

[7] V.V. Afanasjev, B.A. Malomed, and P.L. Chu, "Stability of bound states of pulses in the Ginzburg-Landau equations," Phys. Rev. E, vol. 56, no. 5, pp. 6020-6025, Nov. 1997.

[8] D.Y. Tang, B. Zhao, L.M. Zhao, and H.Y. Tam, "Soliton interaction in a fiber ring laser," Phys. Rev. E, vol. 72, pp. 016616 , Jul. 2005.

[9] Ph. Grelu, F. Belhache, F. Gutty, and J.-M. Soto-Crespo, 'Phaselocked soliton pairs in a stretched-pulse fiber laser," Opt. Lett., vol. 27, no. 11, pp. 966-968, Jun. 2002.

[10] A. Komarov, A. Haboucha, and F. Sanchez,'Ultrahigh-repetitionrate bound-soliton harmonic passive mode-locked fiber lasers," Opt. Lett., vol. 33, no. 19, pp. 2254-2256, Oct. 2008.

[11] A. Komarov, K. Komarov, and F. Sanchez, "Quantization of binding energy of structural solitons in passive mode-locked fiber lasers," Phys. Rev. A, vol. 79, pp. 033807, Mar. 2009.

[12] J.-M. Soto-Crespo, N. Akhmediev, Ph. Grelu, and F. Belhache, "Quantized separations of phase-locked soliton pairs in fiber lasers," Opt. Lett., vol. 28, no. 19, pp. 1757-1759, Oct. 2003.

[13] X. Wu, D.Y. Tang, X.N. Luan, and Q. Zhang, "Bound states of solitons in a fiber laser mode locked with carbon nanotube saturable absorber," Opt. Commun., vol. 284, pp. 3615-3618, Apr. 2011.

[14] J.-L. Xu, X.-L. Li, Y.-Z. Wu, X.-P. Hao, J.-L. He, and K.-J. Yang, "Graphene saturable absorber mirror for ultra-fast-pulse solid-state laser," Opt. Lett., vol. 36, no. 10, pp. 1948-1950, May 2011.

[15] K.P. Komarov, "Theory of stationary ultrashort pulses in solidstate lasers with passive mode locking,"Optics and Spectroscopy, vol. 60, no. 2, pp. 231-234, Feb. 1986. 